Bull. Austral. Math. Soc.

34B15, 34B16, 34B18

VOL. 73 (2006) [175-182]

\title{
MULTIPLICITY OF POSITIVE PERIODIC SOLUTIONS TO SECOND ORDER DIFFERENTIAL EQUATIONS
}

\author{
Jifeng Chu, Xiaoning Lin, Daqing Jiang, Donal O'Regan, R.P. Agarwal
}

In this paper, we study the existence of positive periodic solutions to the equation $x^{\prime \prime}=f(t, x)$. It is proved that such a equation has more than one positive periodic solution when the nonlinearity changes sign. The proof relies on a fixed point theorem in cones.

\section{INTRODUCTION}

In this paper, we are concerned with the existence of single and multiple (strictly) positive 1-periodic solutions to the equation

$$
x^{\prime \prime}=f(t, x),
$$

where $f(t, x): \mathbf{R} \times(0, \infty) \rightarrow \mathbf{R}$ is continuous and 1-periodic in the first variable. By a positive periodic solution of $(1.1)$ we understand a function $x \in C([0,1],(0, \infty))$ satisfying (1.1) and the periodic boundary condition

$$
x(0)=x(1), \quad x^{\prime}(0)=x^{\prime}(1) .
$$

The existence of positive periodic solutions to equation (1.1) has been extensively studied in the literature (see, for example, $[1,2,3]$ and the references therein). In these papers, the two most common techniques to establish existence are the theory of upper and lower solutions [4] and topological degree theory [5]. On the other hand, some fixed point theorems in cones for completely continuous operators have been extensively employed in studying the existence of positive solutions to boundary value problems [6]. However, for the periodic problem, a theory using cones has only recently [7] been applied. One of the difficulties involved in discussing the periodic problem is the sign of the Green's functions for the corresponding linear periodic problem. In [7], Torres succeeded in overcoming this difficulty by using a new $L^{p}$-anti-maximum principle and obtained some new existence results for problem (1.1)-(1.2) by a well known fixed point theorem of compression and expansion of cones.

The aim of this paper is to use some of the basic results in [7] together with a new fixed point theorem in cones to obtain the existence of single and multiple positive periodic solutions to (1.1). The results we obtain are new.

Received 30th August, 2005

Copyright Clearance Centre, Inc. Serial-fee code: 0004-9727/06 \$A2.00+0.00. 


\section{Preliminaries}

Let $a(t)$ be a 1-periodic function and $a \in L^{1}(0,1)$. Now we consider the linear equation

$$
x^{\prime \prime}+a(t) x=0
$$

with the periodic boundary condition (1.2). In this section, we assume conditions under which the only solution to equation (2.1)-(1.2) is the trivial one. As a result, the nonhomogeneous problem

$$
x^{\prime \prime}+a(t) x=h(t), \quad x(0)=x(1), \quad x^{\prime}(0)=x^{\prime}(1)
$$

has a unique solution given by

$$
x(t)=(\mathcal{L} h)(t):=\int_{0}^{l} G(t, s) h(s) d s .
$$

Here $G(t, s)$ is the Green function. Let us define

$$
\Lambda^{-}=\{a \prec 0\}, \quad \Lambda^{+}=\left\{a \succ 0,\|a\|_{p}<K(2 q) \text { for some } 1 \leqslant p \leqslant+\infty\right\} .
$$

Here the notation $a \succ 0$ means that $a(t) \geqslant 0$ for all $t \in[0,1]$ and $a(t)>0$ for $t$ in a subset of positive measure, $a \prec 0$ means that $-a \succ 0$ and $\|\cdot\|_{p}$ denotes the usual $L^{p}$-norm over $(0,1)$ for any given exponent $p \in[1, \infty]$. The conjugate exponent of $p$ is denoted by $q$ : $1 / p+1 / q=1$. The explicit formula for $\mathbf{K}(q)$ is

$$
\mathbf{K}(q)= \begin{cases}\frac{2 \pi}{q}\left(\frac{2}{2+q}\right)^{1-2 / q}\left(\frac{\Gamma(1 / q)}{\Gamma(1 / 2+1 / q)}\right)^{2} & \text { if } 1 \leqslant q<\infty \\ 4 & \text { if } q=\infty\end{cases}
$$

where $\Gamma$ is the Gamma function. Now we present two basic results which were established by Torres in [7].

LEMMA 2.1. ([7]) Assume that $a(t) \in \Lambda^{-}$, then $G(t, s)<0$ for all $(t, s) \in[0,1]$ $\times[0,1]$.

LEMMA 2.2. ([7]) Assume that $a(t) \in \Lambda^{+}$, then $G(t, s)>0$ for all $(t, s) \in[0,1]$ $\times[0,1]$.

REMARK 2.3. If $p=1$, condition $\|a\|_{p}<K(2 q)$ can be weakened to $\|a\|_{1} \leqslant K(\infty)=4$ by the celebrated stability criterion of Lyapunov. In case $p=\infty$, condition $\|a\|_{p}<\mathbf{K}(2 q)$ reads as $\|a\|_{\infty}<\mathbf{K}(2)=\pi^{2}$, which is a well known criterion for the anti-maximum principle used in related literature. In this case, $\|a\|_{p}<\mathbf{K}(2 q)$ can be weakened to $a(t) \prec \pi^{2}$.

In the following, we always denote

$m=\min _{0 \leqslant s, t \leqslant 1} G(t, s), M=\max _{0 \leqslant s, t \leqslant 1} G(t, s), \sigma=m / M$ if $a(t) \in \Lambda^{+}$and $\sigma=M / m$ if $a(t) \in \Lambda^{-}$. 
Thus $M>m>0$ if $a(t) \in \Lambda^{+}$and $m<M<0$ if $a(t) \in \Lambda^{-}$. In either case, we have $0<\sigma<1$.

In this paper we shall establish the existence of positive periodic solutions to equation (1.1), using the following well known fixed point theorem in cones [8].

TheOREM 2.4. Let $X$ be a Banach space and $K$ be a cone in $X$. Assume $\Omega_{1}, \Omega_{2}$ are open subsets of $X$ with $0 \in \Omega_{1}, \bar{\Omega}_{1} \subset \Omega_{2}$. Let

$$
\Phi: K \cap\left(\bar{\Omega}_{2} \backslash \Omega_{1}\right) \rightarrow K
$$

be a continuous and completely continuous operator such that

(i) $\|\Phi x\| \leqslant\|x\|$ for $x \in K \cap \partial \Omega_{1}$,

(ii) there exists $\psi \in K \backslash\{0\}$ such that $x \neq \Phi x+\lambda \psi$ for $x \in K \cap \partial \Omega_{2}$ and $\lambda>0$. Then $\Phi$ has a fixed point in $K \cap\left(\bar{\Omega}_{2} \backslash \Omega_{1}\right)$.

REMARK 2.5. In Theorem 2.4, if (i) and (ii) are replaced by

(i)* $\|\Phi x\| \leqslant\|x\|$ for $x \in K \cap \partial \Omega_{2}$, and

(ii)* there exists $\psi \in K \backslash\{0\}$ such that $x \neq \Phi x+\lambda \psi$ for $x \in K \cap \partial \Omega_{1}$ and $\lambda>0$, then $\Phi$ has also a fixed point in $K \cap\left(\bar{\Omega}_{2} \backslash \Omega_{1}\right)$.

In applications below, we take $X=C[0,1]$ with the supremum norm $\|\cdot\|$ and define

$$
K=\left\{x \in X: x(t) \geqslant 0 \text { for all } t \text { and } \min _{0 \leqslant t \leqslant 1} x(t) \geqslant \sigma\|x\|\right\},
$$

where $\sigma$ is as in (2.6).

One may readily verify that $K$ is a cone in $X$. Suppose now that $F:[0,1] \times \mathbf{R}$ $\rightarrow[0, \infty)$ is a continuous function and define an operator $T: X \rightarrow X$ by

$$
(T x)(t)=\int_{0}^{1} G(t, s) F(s, x(s)) d s
$$

for $x \in X$ and $t \in[0,1]$. It is easy to prove:

Lemma 2.6. $T$ is well defined and maps $X$ into $K$. Moreover, $T$ is continuous and completely continuous.

\section{MAIN RESUltS}

In this section we establish the existence and multiplicity of positive periodic solutions to (1.1).

THEOREM 3.1. Suppose that there exist $a \in \Lambda^{+}$and $0<r<R$ such that

$$
f(t, x)+a(t) x \geqslant 0, \quad \forall x \in[\sigma r, R] .
$$

Then Equation (1.1) has at least one positive solution if one of the following two conditions holds 
(I) $f(t, x) \geqslant 0, \quad \forall x \in[\sigma r, r]$ and $f(t, x) \leqslant 0, \quad \forall x \in[\sigma R, R]$;

(II) $f(t, x) \leqslant 0, \quad \forall x \in[\sigma r, r]$ and $f(t, x) \geqslant 0, \quad \forall x \in[\sigma R, R]$.

Proof: The existence is established using Theorem 2.4 and Remark 2.5. To do so, let us write equation (1.1) as

$$
x^{\prime \prime}+a(t) x=f(t, x)+a(t) x .
$$

Define the open sets

$$
\Omega_{r}=\{x \in C[0,1]:\|x\|<r\} \text { and } \Omega_{R}=\{x \in C[0,1]:\|x\|<R\} .
$$

Let $K$ be a cone defined by (2.7) and define an operator on $K$ by

$$
(\Phi x)(t)=\int_{0}^{1} G(t, s)[f(s, x(s))+a(t) x] d s .
$$

Clearly, $\Phi: K \cap\left(\bar{\Omega}_{R} \backslash \Omega_{r}\right) \rightarrow C[0,1]$ is continuous and completely continuous since $f:[0,1] \times[\sigma r, R] \rightarrow R$ is continuous. Also we have $\Phi(K) \subset K$.

Let us suppose that condition (I) holds (the proof for condition (II) is similar).

By the first inequality of condition (I), we have $f(t, x)+a(t) x \geqslant a(t) x, \forall x \in[\sigma r, r]$. Let $\psi \equiv 1$, so $\psi \in K$. Now we prove that

$$
x \neq \Phi x+\lambda \psi, \quad \forall x \in K \cap \partial \Omega_{r} \text { and } \lambda>0 .
$$

Suppose not, that is, suppose there exist $x_{0} \in K \cap \partial \Omega_{r}$ and $\lambda_{0}>0$ such that $x_{0}$ $=\Phi x_{0}+\lambda_{0} \psi$. Now since $x_{0} \in K \cap \partial \Omega_{r}$, then $x_{0}(t) \geqslant \sigma\left\|x_{0}\right\|=\sigma r$. Let $\mu=\min _{t \in[0,1]} x_{0}(t)$. Then we have

$$
\begin{aligned}
x_{0}(t) & =\left(\Phi x_{0}\right)(t)+\lambda_{0}=\int_{0}^{1} G(t, s)\left[f\left(s, x_{0}(s)\right)+a(t) x_{0}(s)\right] d s+\lambda_{0} \\
& \geqslant \int_{0}^{1} G(t, s) a(s) x_{0}(s) d s+\lambda_{0} \geqslant \mu \int_{0}^{1} G(t, s) a(s) d s+\lambda_{0}=\mu+\lambda_{0} .
\end{aligned}
$$

This implies $\mu \geqslant \mu+\lambda_{0}$, a contradiction. Therefore, (3.3) holds.

On the other hand, by the second inequality of condition (I), we have

$$
f(t, x)+a(t) x \leqslant a(t) x, \quad \forall x \in[\sigma R, R] .
$$

Now we prove that

$$
\|\Phi x\| \leqslant\|x\|, \quad \forall x \in K \cap \partial \Omega_{R} .
$$

In fact, for any $x \in K \cap \partial \Omega_{R}$, we have

$$
\begin{aligned}
(\Phi x)(t) & =\int_{0}^{1} G(t, s)[f(s, x(s))+a(t) x] d s \leqslant \int_{0}^{1} G(t, s) a(s) x(s) d s \\
& \leqslant \int_{0}^{1} G(t, s) a(s) d s \cdot \max _{t \in[0,1]} x(t)=\|x\| .
\end{aligned}
$$


Therefore, $\|\Phi x\| \leqslant\|x\|$, that is, (3.4) holds.

It follows from Remark $2.5,(3.3)$ and (3.4) that $\Phi$ has a fixed point $x \in K \cap\left(\bar{\Omega}_{R} \backslash \Omega_{\mathrm{r}}\right)$. Clearly, this fixed point is a positive solution of (1.1) satisfying $r \leqslant\|x\| \leqslant R$.

REMARK 3.2. In [7, Theorem 3.2], it is proved that equation (1.1) has at least one positive periodic solution provided one of the following two conditions holds for some $a(t) \in \Lambda^{+}$and $0<r<R$ :

$(\mathrm{I})^{*} \quad f(t, x)+a(t) x \geqslant\left(M / m^{2}\right) x, \forall x \in[(m / M) r, r] ; \quad f(t, x)+a(t) x \leqslant 1 / M$, $\forall x \in[R,(M / m) R]$

(II) $)^{*} \quad f(t, x)+a(t) x \leqslant 1 / M, \forall x \in[(m / M) r, r] ; \quad f(t, x)+a(t) x \geqslant\left(M / m^{2}\right) x$, $\forall x \in[R,(M / m) R]$.

Theorem 3.1 improves the above result since we only need the sign of $f(t, x)$ in (I) and (II).

The following multiplicity result follows immediately from Theorem 3.1.

THEOREM 3.3. Suppose that there exist $a \in \Lambda^{+}$and $0<r<p<R$ such that

$$
f(t, x)+a(t) x \geqslant 0, \quad \forall x \in[\sigma r, R] .
$$

Then Equation (1.1) has at least two positive periodic solutions if one of the following two conditions holds

(I) $f(t, x) \geqslant 0, \quad \forall x \in[\sigma r, r] ; \quad f(t, x)<0, \quad \forall x \in[\sigma p, p] ; \quad f(t, x) \geqslant 0$, $\forall x \in[\sigma R, R]$

(II) $f(t, x) \leqslant 0, \quad \forall x \in[\sigma r, r] ; \quad f(t, x)>0, \quad \forall x \in[\sigma p, p] ; \quad f(t, x) \leqslant 0$, $\forall x \in[\sigma R, R]$.

Proof: We only prove the result when condition (I) holds. Define $\Omega_{r}, \Omega_{R}, K$ and $\Phi$ as in Theorem 3.1 and define $\Omega_{p}=\{x \in C[0,1]:\|x\|<p\}$.

Essentially the same reasoning as in the proof of Theorem 3.1 guarantees that

$$
\begin{gathered}
x \neq \Phi x+\lambda \psi \text { for } \forall x \in K \cap \partial \Omega_{r} \text { and } \lambda>0 ; \\
x \neq \Phi x+\lambda \psi \text { for } \forall x \in K \cap \partial \Omega_{R} \text { and } \lambda>0 ; \\
\|\Phi x\|<\|x\| \text { for } \forall x \in K \cap \partial \Omega_{p} .
\end{gathered}
$$

Thus we can obtain the existence of two positive solutions $x_{1}$ and $x_{2}$ by using Theorem 2.4 and Remark 2.5 once, respectively. It is easy to see that $r \leqslant\left\|x_{1}\right\|<p<\left\|x_{2}\right\| \leqslant R$ since (3.7) holds.

Next we consider the case of $a(t) \in \Lambda^{-}$. Here we only state the results and omit their proofs since they can be proved in a similar way to that of Theorems 3.1 and 3.3.

THEOREM 3.4. Suppose that there exist $a \in \Lambda^{-}$and $0<r<R$ such that

$$
f(t, x)+a(t) x \leqslant 0, \quad \forall x \in[\sigma r, R] .
$$


Then Equation (1.1) has at least one positive periodic solution if one of the following two conditions holds

(I) $f(t, x) \geqslant 0, \quad \forall x \in[\sigma r, r]$ and $f(t, x) \leqslant 0, \quad \forall x \in[\sigma R, R]$;

(II) $f(t, x) \leqslant 0, \quad \forall x \in[\sigma r, r]$ and $f(t, x) \geqslant 0, \quad \forall x \in[\sigma R, R]$.

THEOREM 3.5. Suppose that there exist $a \in \Lambda^{-}$and $0<r<p<R$ such that

$$
f(t, x)+a(t) x \leqslant 0, \quad \forall x \in[\sigma r, R] .
$$

Then Equation (1.1) has at least two positive periodic solutions if one of the following two conditions holds

$$
\begin{array}{ll}
\text { (I) } & f(t, x) \geqslant 0, \quad \forall x \in[\sigma r, r] ; \quad f(t, x)<0, \quad \forall x \in[\sigma p, p] ; f(t, x) \geqslant 0, \\
& \forall x \in[\sigma R, R] ; \\
\text { (II) } f(t, x) \leqslant 0, \quad \forall x \in[\sigma r, r] ; \quad f(t, x)>0, \quad \forall x \in[\sigma p, p] ; f(t, x) \leqslant 0, \\
\\
\forall x \in[\sigma R, R] .
\end{array}
$$

REMARK 3.6 In fact, we can obtain the existence of more than two positive periodic solutions of equation (1.1) provided $f(t, x)$ satisfies the required inequalities.

EXAMPLE 3.7. Let us consider the following nonsingular equation

$$
x^{\prime \prime}+a(t) x=\mu b(t)\left(x^{\alpha}+x^{\beta}\right)
$$

where $0<\alpha<1<\beta, a \in C[0,1], b \in C[0,1]$ is a positive function, $a(t) \in \Lambda^{+}$and $\mu>0$ is a positive parameter. Then equation (3.8) has at least two positive periodic solutions for each $0<\mu<\mu^{*}$, where $\mu^{*}$ is a positive constant described below.

To show this we shall apply Theorem 3.3 with $f(t, x)=\mu b(t)\left(x^{\alpha}+x^{\beta}\right)-a(t) x$. It is easy to see that

$$
\lim _{x \rightarrow 0^{+}} \frac{f(t, x)}{x}=+\infty \text { and } \lim _{x \rightarrow+\infty} \frac{f(t, x)}{x}=+\infty
$$

since $0<\alpha<1<\beta$. Set

$$
T(x)=\frac{x}{x^{\alpha}+x^{\beta}}, \quad x>0 .
$$

Then $T\left(0^{+}\right)=T(\infty)=0$ and

$$
T(x) \leqslant T(p)=\sup _{x \in(0, \infty)} T(x), \text { where } p=\left(\frac{1-\alpha}{\beta-1}\right)^{1 /(\beta-\alpha)} .
$$

Let $\mu^{*}=\sigma T(p) e^{-1}$, where $e=\max _{t \in[0,1]}(b(t)) /(a(t))$. Then for $x \in[\sigma p, p]$, we have

$$
\begin{aligned}
f(t, x) & =\mu b(t)\left(x^{\alpha}+x^{\beta}\right)-a(t) x<\mu^{*} a(t)\left(p^{\alpha}+p^{\beta}\right) \max _{t \in[0,1]} \frac{b(t)}{a(t)}-a(t) \sigma p \\
& =\sigma T(p) a(t)\left(p^{\alpha}+p^{\beta}\right)-a(t) \sigma p=0 .
\end{aligned}
$$


(3.9) and (3.10) imply that condition (I) of Theorem 3.3 is satisfied, so the existence is guaranteed.

EXAMPLE 3.8. Let us consider the following singular repulsive equation [7]

$$
x^{\prime \prime}-\frac{a}{x^{\lambda}}+k^{2} x=e(t)
$$

with $a>0, k \in(0, \pi), \lambda>0$ and $e \in C[0,1]$. Let $e^{*}=\max _{t \in[0,1]} e(t)$ and $e_{*}=\min _{t \in[0,1]} e(t)$. Then

(i) Equation (3.11) has at least one positive periodic solution for each $e(t)$ with $e_{*} \geqslant 0$; and

(ii) Equation (3.11) has at least one positive periodic solution for each $e(t)$ with $e_{*}<0$ and satisfying the following inequality:

$$
e^{*} \leqslant \frac{e_{*}}{\cos ^{\lambda}(k / 2)}+k^{2}\left(\frac{a}{\left|e_{*}\right|}\right)^{1 / \lambda} \cos (k / 2) .
$$

If $k \in(0, \pi)$, then $k \in \Lambda^{+}$and we can obtain the following explicit values (see [7])

$$
m=\frac{1}{2 k} \cot \left(\frac{k}{2}\right), \quad M=\frac{1}{2 k \sin (k / 2)} \text { and } \sigma=\cos \left(\frac{k}{2}\right) .
$$

Now (i) is a direct result of Theorem 3.1 since $f(t, x)=a /\left(x^{\lambda}\right)+e(t)-k^{2} x \rightarrow+\infty$ as $x \rightarrow 0$ and $f(t, x) \rightarrow-\infty$ as $x \rightarrow+\infty$.

Next we prove (ii). Condition (I) of Theorem 3.1 reduces to finding $R>0$ such that

$$
\frac{a}{x^{\lambda}}+e_{*} \geqslant 0, \quad \forall x \in(0, R]
$$

and

$$
\frac{a}{x^{\lambda}}+e^{*} \leqslant k^{2} x, \quad \forall x \in\left[R \cos \left(\frac{k}{2}\right), R\right]
$$

Now, we fix $R=\left(a /\left|e_{*}\right|\right)^{1 / \lambda}$, then inequality (3.13) is satisfied. By using the monotonocity of $k^{2} x-\left(a / x^{\lambda}\right)$, then (3.14) holds if

$$
e^{*} \leqslant \frac{e_{*}}{\cos ^{\lambda}(k / 2)}+k^{2}\left(\frac{a}{\left|e_{*}\right|}\right)^{1 / \lambda} \cos (k / 2) .
$$

REMARK 3.9. In [7], it is proved that equation (3.11) has at least one positive periodic solution if $k \in(0, \pi), e \in L^{\infty}[0,1], e_{*}<0$ and the following inequality holds:

$$
e^{*} \leqslant \frac{e_{*}}{\cos ^{\lambda}(k / 2)}+k\left(a /\left|e_{*}\right|\right)^{1 / \lambda} \sin (k) \text {. }
$$

It is easy to see that our condition (3.12) is weaker than condition (3.15) since

$$
k^{2}\left(\frac{a}{\left|e_{*}\right|}\right)^{1 / \lambda} \cos \left(\frac{k}{2}\right) \geqslant k\left(\frac{a}{\left|e_{*}\right|}\right)^{1 / \lambda} \sin (k), \quad \forall k \in(0, \pi) .
$$




\section{REFERENCES}

[1] A.C. Lazer and S. Solimini, 'On periodic solutions of nonlinear differential equations with singularities', Proc. Amer. Math. Soc. 99 (1987), 109-114.

[2] I. Rachunkova, M. Trrdý and I. Vrkox, 'Existence of nonnegative and nonpositive solutions for second order periodic boundary value problems', J. Differential Equations 176 (2001), 445-469.

[3] M.R. Zhang, 'A relationship between the periodic and the Dirichlet BVPs of singular differential equations', Proc. Roy. Soc. Edinburgh Sect. A 128 (1998), 1099-1114.

[4] C. De Coster and P. Habets, 'Upper and lower solutions in the theory of ODE boundary value problems: classical and recent results', in Nonlinear Analysis and Boundary Value Problems for Ordinary Differential Equations, CISM-ICMS 371, (F. Zano-lin, Editor) (Springer-Verlag, New York, 1996), pp. 1-78.

[5] J. Mawhin, 'Topological degree and boundary value problems for nonlinear differential equations', in Topological Methods for Ordinary Differential Equations, (M. Furi and P. Zecca, Editors), Lecture Notes Math. 1537 (Springer-Verlag, New York, Berlin, 1993), pp. 74-142.

[6] L.H. Erbe and R.M. Mathsen, 'Positive solutions for singular nonlinear boundary value problems', Nonlinear Anal. 46 (2001), 979-986.

[7] P.J. Torres, 'Existence of one-signed periodic solutions of some second-order differential equations via a Krasnoselskii fixed point theorem', J. Differential Equations 190 (2003), 643-662.

[8] K. Deimling, Nonlinear functional analysis (Springer-Verlag, Berlin, 1985).

Department of Mathematical Sciences

Tsinghua University

Beijing 100084

China

Department of Mathematics

Northeast Normal University

Changchun 130024, Jilin

China

Department of Mathematical Science

Florida Institute of Technology

Melbourne, FL 32901-6975

United States of America
Department of Mathematics

Northeast Normal University

Changchun 130024, Jilin

China

Department of Mathematics

National University of Ireland

Galway

Ireland 